\title{
NONLINEAR FOURIER ANALYSIS
}

\author{
STEPHEN SEMMES
}

What is nonlinear Fourier Analysis? Let us consider an example.

Let $\Omega$ be a domain in the plane bounded by a Jordan curve $\Gamma$ that goes to $\infty$. Let $\Phi$ be a Riemann mapping of the upper half-plane onto $\Omega$.

How does $\Phi$ behave as a function of $\Gamma$ ? Is it continuous? smooth? realanalytic? Let's think of ourselves as calculus students; we are presented with this function, and we want to study its basic properties.

Of course, the Riemann mapping is not uniquely determined by the curve, and we have to do something about that. Let's ignore that issue for the moment.

There is a more serious problem with the question we've raised. To talk about the continuity of this function, we need to specify a domain of definition for it, the range space for its values, and topologies for both. To consider smoothness or real-analyticity, we need more structure on both the domain and range.

This brings us to another component of the above question: What are the most natural choices for the domain and range? We would like the domain to be as large as possible so that the function is defined and well behaved. In this case, the domain is a space of curves, and we want in particular to minimize the smoothness assumptions on the curves. We would like for the domain to be a space of curves characterized by some natural geometric condition.

There is a beautiful theorem of Coifman and Meyer [CM3] that says that the Riemann mapping is a real-analytic function on a natural space of curves, and with a natural choice of range space. It is a bit technical to state it precisely now, but I shall say more about it at the end of this section.

The problem of understanding the Riemann mapping as a function of the curve is a good example of a problem in nonlinear Fourier analysis. There are many basic objects in mathematics which can be viewed naturally as nonlinear functions in infinite dimensions, and we want to study their basic properties as such.

There are also cases in which we are forced to confront such issues of nonlinear dependence even when the original problem does not directly call for it. We shall see examples of this shortly.

The reason that this is nonlinear Fourier analysis, and not just nonlinear analysis, is that we often need Fourier analysis to deal with the objects that

Received by the editors April 15, 1988.

1980 Mathematics Subject Classification (1985 Revision). Primary 42B99.

The author is partially supported by the NSF and the Alfred P. Sloan Foundation. 
arise in these problems and to obtain needed estimates for them. This is particularly true when we try to make optimal choices for the domain and range spaces.

This subject should be viewed very broadly, but the remaining examples described in this first part come from a short list of problems that have been studied extensively by Fourier analysts in recent years. Some of the general ideas for treating problems like these are discussed in the second section. The third section is devoted to a brief description of some aspects of Littlewood-Paley theory, which provides important tools for doing the analysis.

I shall concentrate on explaining examples and general ideas used to treat them at the expense of recent results and more refined techniques. Few details will be included, in the hope of being accessible to as large an audience as possible. Essentially nothing of what I discuss is due to me.

To balance these choices a fourth section is included with a brief guide to some other expository papers of a more technical nature on related topics. Fortunately, Yves Meyer is preparing a book that will provide a basic reference.

The next example that I want to discuss is the Cauchy integral operator $C_{\Gamma}$ on a curve $\Gamma$, viewed as an operator-valued function of the curve. This operator is given by a principal value singular integral: if $f$ is a function on $\Gamma$, we define $C_{\Gamma}(f)$ on $\Gamma$ by

$$
C_{\Gamma} f(z)=\frac{1}{2 \pi i} p v \int_{\Gamma} \frac{f(w)}{w-z} d w, \quad z \in \Gamma .
$$

This operator is probably less familiar than

$$
F(z)=\frac{1}{2 \pi i} \int_{\Gamma} \frac{f(w)}{w-z} d w, \quad z \notin \Gamma,
$$

which is defined and holomorphic on $C \backslash \Gamma$. There is a simple formula that gives the boundary values of $F$ on $\Gamma$ in terms of $f$ and $C_{\Gamma} f$.

The operator $C_{\Gamma}$ also has important but nonobvious connections with the Riemann mapping and PDE. In particular, the dependence of the Riemann mapping on the curve $\Gamma$ is intimately related to the dependence of $C_{\Gamma}$ on $\Gamma$.

As before, we not only want to study the dependence of $C_{\Gamma}$ on $\Gamma$, but we also wish to choose an optimal space of curves on which to work. To simplify the discussion, we shall restrict ourselves to curves that are graphs, and work with a slightly simplified operator.

Given $A: \mathbf{R} \rightarrow \mathbf{R}$, we define an operator $T_{A}$, acting on functions $f$ defined on $\mathbf{R}$, by

$$
T_{A} f(x)=p v \int_{-\infty}^{\infty} \frac{f(y)}{x-y+i(A(x)-A(y))} d y, \quad x \in \mathbf{R} .
$$

This is the Cauchy integral on the graph of $A$ written in terms of the graph parameterization $x \rightarrow x+i A(x)$, except that we dropped a factor of $1+i A^{\prime}(y)$ when we replaced $d z$ by $d y$, and we also threw away a factor of $-1 / 2 \pi i$. 
We need to choose a domain and range for the operator-valued function $A \rightarrow T_{A}$. Let us take the range to be $B\left(L^{2}(\mathbf{R})\right)$, the Banach space of all bounded linear operators on $L^{2}(\mathbf{R})$.

We want to choose the domain of $T_{A}$ to be as large as possible so that it is a smooth function of $A$, or even real-analytic. Let us use the explicit formula for $T_{A}$ to compute its power series about $A=0$. This will help us find what conditions on $A$ are needed for $T_{A}$ to be smooth or real-analytic.

From (1.3) we have

$$
\begin{aligned}
T_{A} f(x) & =p v \int_{-\infty}^{\infty} \frac{1}{x-y}\left(1+i \frac{A(x)-A(y)}{x-y}\right)^{-1} f(y) d y \\
& =\sum_{k=0}^{\infty} p v \int_{-\infty}^{\infty} \frac{1}{x-y}(-i)^{k}\left(\frac{A(x)-A(y)}{x-y}\right)^{k} f(y) d y \\
& =\sum_{k=0}^{\infty} T_{k}(A) f .
\end{aligned}
$$

In order for this series to have any hope to converge, we must have

$$
\left|\frac{A(x)-A(y)}{x-y}\right|<1 \text {. }
$$

Moreover, for each $k \geq 1, T_{k}(A) \in B\left(L^{2}\right)$ implies that $A$ is Lipschitz, i.e., that there is an $M>0$ such that $|A(x)-A(y)| \leq M|x-y|$ for all $x, y \in \mathbf{R}$. Because $T_{1}(B)$ is just the derivative of $A \rightarrow T_{A}$ at $A=0$ in the direction of $B$, this says that for $T_{A}$ to be merely differentiable, we already need to restrict ourselves to functions $A$ that are Lipschitz.

This suggests that the natural domain for $T_{A}$ is the Banach space Lip of all Lipschitz functions on $\mathbf{R}$. (Note that an equivalent formulation of the Lipschitz condition on $A$ is that $A$ should be absolutely continuous and $A^{\prime} \in L^{\infty}(\mathbf{R})$.)

THEOREM 1.5. $T_{A}$ is a bounded operator on $L^{2}(\mathbf{R})$ if $A$ is Lipschitz. Moreover, $A \rightarrow T_{A}$ is a real-analytic map from $\operatorname{Lip}$ to $B\left(L^{2}(\mathbf{R})\right)$.

This theorem was proved by Calderón [C1] when $\left\|A^{\prime}\right\|_{\infty}$ is small, and by Coifman, McIntosh, and Meyer [CMM] in the general case.

I should say something about what real-analyticity means in this context. I won't give a precise definition yet, but it means that the power series in (1.4) should converge absolutely in $B\left(L^{2}\right)$ when $\left\|A^{\prime}\right\|_{\infty}$ is small enough, and that an analogous power series expansion about any other $A_{0} \in$ Lip should converge in a small neighborhood of $A_{0}$. In this particular case the radius of convergence about $A_{0}=0$ is actually 1 .

The first half of Theorem 1.5 is extremely interesting in its own right. The assumption $A \in \mathrm{Lip}$ is very natural; it is very geometrical, involving the right amount of smoothness, and it scales properly.

The fact that $T_{A} \in B\left(L^{2}\right)$ if $A \in$ Lip is completely inaccessible by classical techniques. Because $T_{A}$ is not a convolution operator, standard Fourier transform methods (e.g., Plancherel's theorem) do not apply. Hilbert space 
results like the Cotlar-Stein lemma don't work either. If $A$ were smooth, $T_{A}$ could be expressed in terms of the Hilbert transform $\left(=T_{A}\right.$ when $\left.A=0\right)$ and a simple error term, and the Hilbert transform can be dealt with using Plancherel or Hilbert space methods. When $A$ is merely Lipschitz, this error term is no longer easily tractable.

In other words, when $A$ is not smooth, you cannot simply throw it away; when analyzing $T_{A} f$, you have to treat $A$ on an equal footing with $f$. Thus, although the question "Is $T_{A}$ bounded on $L^{2}$ if $A$ is Lipschitz?" does not superficially seem to involve the problem of the dependence of $T_{A}$ on $A$, this issue does arise when you try to answer the question.

For example, it is natural to try to prove that $T_{A} \in B\left(L^{2}\right)$ for $\left\|A^{\prime}\right\|_{\infty}<1$ by showing that each $T_{k}(A) \in B\left(L^{2}\right)$ with norms that add up. Such an approach would automatically give the real-analyticity of $T_{A}$ also.

It turns out that the real-analyticity of this particular operator-valued function $T_{A}$ is a soft and painless consequence of the fact that $T_{A} \in B\left(L^{2}\right)$ if $A \in \mathrm{Lip}$. Thus, it is not only natural to consider the dependence of $T_{A}$ on $A$ when trying to prove that $T_{A} \in B\left(L^{2}\right)$, but in a very real sense the two are the same problem.

The third example I want to discuss is the general problem of studying how the solutions of a PDE behave as nonlinear functions of the coefficients.

Let us consider a specific case. Define a differential operator $L$ on $\mathbf{R}^{n}$ by

$$
L=\sum_{i j} b_{i j}(x) \frac{\partial^{2}}{\partial x_{i} \partial x_{j}},
$$

and let us assume that the coefficients are bounded and elliptic:

$$
\delta|\zeta|^{2} \leq \sum b_{i j}(x) \zeta_{i} \zeta_{j} \leq C|\zeta|^{2} \forall \zeta \in \mathbf{R}^{n},
$$

where $0<\delta<C<\infty$.

Consider solutions of the initial-value problem for the heat equation associated to $L$,

$$
\frac{\partial u}{\partial t}=L u, \quad u=u(x, t), \quad u(x, 0)=f(x) .
$$

We can view $u$ as a function of both $B=\left(b_{i j}(x)\right)$ and $f$, and it is linear in $f$. How does it behave as a function of $B$ ?

We can rewrite $u$ as

$$
u(\cdot, t)=e^{t L} f(\cdot) .
$$

Thus the previous question is equivalent to asking how $e^{t L}$ behaves as an operator-valued function of $B$. More generally, we can ask for which functions $F$ it is true that $F(L)$ is defined and real-analytic in $B$.

Let me describe a closely related problem, often called Kato's problem by harmonic analysts, or the square-root problem by operator-theorists. Let $A=\left(a_{i j}(x)\right)$ be a matrix-valued function on $\mathbf{R}^{n}$ that is bounded, measurable, and accretive. This last means that

$$
\operatorname{Re}\langle A(x) \zeta, \zeta\rangle \geq \delta|\zeta|^{2}
$$


for some $\delta>0$ and all $x, \zeta \in \mathbf{R}^{n}$. In this case $(\operatorname{div} A \nabla)^{1 / 2}$ can be defined in a natural way, where

$$
\operatorname{div} A \nabla=\sum_{i j} \frac{\partial}{\partial x_{i}} a_{i j}(x) \frac{\partial}{\partial x_{j}}
$$

The problem is whether the domain of $(\operatorname{div} A \nabla)^{1 / 2}$ is the Sobolev space $H^{1}$, i.e., whether

$$
(\operatorname{div} A \nabla)^{1 / 2} f \in L^{2} \quad \text { if } \nabla f \in L^{2} .
$$

If $A$ is selfadjoint, then this follows from spectral theory, but that doesn't work in the general case. In fact, estimates for (1.7) in the general case imply real-analyticity results for $A \rightarrow(\operatorname{div} A \nabla)^{1 / 2}$, which are not obtainable from spectral theory even in the selfadjoint case.

When $n=1,(1.7)$ is true and it was proved by Coifman, McIntosh, and Meyer [CMM] in the same paper where they proved that the Cauchy integral on a Lipschitz graph is bounded on $L^{2}$. The two problems are very closely related; the Cauchy integral on the graph of $A: \mathbf{R} \rightarrow \mathbf{R}$ turns out to be the same as $F\left(\left(1 /\left(1+i A^{\prime}\right)\right)(1 / i)(d / d x)\right)$, where $F(z)=\operatorname{sgn}(\operatorname{Re} z)$.

When $n>1,(1.7)$ has been proved so far only in the case where $\|A-I\|_{\infty}$ is small enough.

The situation is similar for the first problem, concerning functions of $L, L$ as in (1.6). As with (1.7), there is a close correspondence between real-analyticity results for $F(L)$ and the problem of having estimates for $F(L)$ if we allow the coefficients of $L$ to be complex-valued. When $n=1$, the situation is under control as soon as the coefficients are bounded and accretive. When $n>1$, only the case where $\|B-I\|_{\infty}$ is small is under control.

(References to these and related results and applications to PDE include [CM4, CDM, CMM, FJK 1, 2, 3, KM, DJ].)

Thus when $n>1$, we do not know the correct domain of definition for $(\operatorname{div} A \nabla)^{1 / 2}$ or for functions of $L$. We know that it contains a small neighborhood in $L^{\infty}\left(\mathbf{R}^{n}\right)$ about $I$, but we don't know if these domains should be the entire set of bounded, accretive matrix-valued functions. These partial results are still quite interesting though: they tell us that $L^{\infty}$ is the correct Banach space in which the domains should live.

Let me put this differently. Before we can talk about the domain of definition of a nonlinear function in infinite dimensions, we must first choose a vector space (preferably a Banach space) in which the domain should be an open subset. Before we can worry about choosing the domain to be as large as possible, we have to first choose this containing Banach space to be as large as possible.

Thus in the preceding two examples the correct Banach space is the space of bounded measurable matrix-valued functions on $\mathbf{R}^{n}$. In these two cases the correct domain of definition is not known. For the operatorvalued function $T_{A}$, the correct Banach space is Lip, and the correct domain is all of Lip. 
In each of these examples the Banach space containing the natural domain of definition is a classical Banach space like $L^{\infty}$. There are many natural examples where this is not the case. One such example is the true Cauchy integral, given in (1.1), rather than the simpler $T_{A}$ given in (1.3). Let me describe what happens in this case.

Let $\Gamma$ be an oriented rectifiable Jordan curve in the plane that goes to $\infty$. Let us assume that $0 \in \Gamma$, and let $z: \mathbf{R} \rightarrow \Gamma$ be the arclength parameterization that satisfies $z(0)=0$. Given a function $f$ on $\mathbf{R}$, define

$$
\tilde{C}_{\Gamma} f(x)=\frac{1}{2 \pi i} P V \int_{-\infty}^{\infty} \frac{f(z(y))}{z(y)-z(x)} z^{\prime}(y) d y, \quad x \in \mathbf{R} .
$$

This is simply the Cauchy integral operator (1.1) brought back to the real line using the arclength parameterization of $\Gamma$.

We can view $\tilde{C}_{\Gamma}$ as a nonlinear operator-valued function of the curve $\Gamma$. Let us take $B\left(L^{2}(\mathbf{R})\right)$ as our range space, and ask for a natural space of curves on which this function is continuous, smooth, or even real-analytic.

It turns out that the natural space of curves is the space of chord-arc curves. We say that $\Gamma$ is a chord-arc curve with constant $k$ if

$$
|x-t| \leq(1+k)|z(s)-z(t)|
$$

for all $s, t \in \mathbf{R}$. Geometrically, we think of $|z(s)-z(t)|$ as the length of the chord joining $z(s)$ and $z(t)$, while $|s-t|$ is the length of the arc that joins them. The chord-arc condition keeps the curve from smashing into itself. For example, it forbids cusps.

Let us see how the space of chord-arc curves can be naturally identified with an open subset of $\mathrm{BMO}(\mathbf{R})$. A locally integrable function $f$ on $R$ is said to lie in $\mathrm{BMO}(\mathbf{R})(\mathrm{BMO}=$ bounded mean oscillation $)$ if

$$
\|f\|_{*}=\sup _{I} \frac{1}{|I|} \int_{I}\left|f(x)-f_{I}\right| d x<\infty,
$$

where the sup is taken over all intervals $I$, and $f_{I}=\frac{1}{|T|} \int_{I} f$.

Clearly, $L^{\infty} \subseteq \mathrm{BMO}$, but the inclusion is proper, because $\log |x|$ lies in $\mathrm{BMO}$. As the BMO norm indicates, BMO functions are really defined only up to an additive constant.

BMO should be thought of as a close relative of $L^{\infty}$. There are many problems where you might naively hope that $L^{\infty}$ is the correct space to work with, but for which that isn't true, and instead BMO is the right one. This is almost always the case when adding a constant to the function does not change the problem. Of course, this has to happen if BMO is the correct space to be working with.

There is an open subset $\Omega$ in the space of real-valued BMO functions such that $\Gamma$ is a chord-arc curve if and only if $z^{\prime}(t)=e^{i b(t)}$ for some $b \in \Omega$. (For example, $b(t)=\alpha \log |t|, \alpha \in \mathbf{R}$, corresponds to logarithmic spirals.) This gives a topology for the space of chord-arc curves. With this topology, the map $\Gamma \rightarrow \tilde{C}_{\Gamma}$ of $\Omega$ into $B\left(L^{2}\right)$ is a homeomorphism onto its image. Thus this BMO topology on the space of chord-arc curves is 
not only natural, but it is forced on us by the operator theory. The map $\Gamma \rightarrow \tilde{C}_{\Gamma}$ is also real-analytic and locally bilipschitz.

We mentioned above that BMO functions are only defined up to an additive constant. If we add a constant to $b(t)$ and define $\Gamma$ as in the preceding paragraph, then $\Gamma$ is merely rotated, and the operator $\tilde{C}_{\Gamma}$ does not change at all. Thus the mapping of $b \in \Omega$ to $\tilde{C}_{\Gamma}$ is well defined for $b \in \mathrm{BMO}$. As mentioned above, this extra symmetry is what suggests that $\mathrm{BMO}$ is the correct space. By contrast, $A \rightarrow T_{A}$ defined in (1.3) does not have this symmetry, and the corresponding space of curves-i.e., Lipschitz graphs-is not rotationally invariant.

This space of chord-arc curves also shows up in the theorem of Coifman and Meyer on the real-analyticity of the Riemann mapping as a function of the curve. Given an oriented chord-arc curve $\Gamma$, let $D$ denote the domain bounded by $\Gamma$ that lies to the left of $\Gamma$. Let $\Psi$ be a conformal mapping on $D$ onto the upper half-plane. (Thus $\Psi^{-1}$ is a Riemann mapping.) Then $\Psi$ extends continuously to $\Gamma$, and so $h=\Psi \circ z$ defines an increasing homeomorphism on $\mathbf{R}$. A theorem of Lavrentiev implies that $\log h^{\prime} \in \mathrm{BMO}(\mathbf{R})$ if $\Gamma$ is a chord-arc curve. The theorem of Coifman and Meyer states that the map $\Gamma \rightarrow \log h^{\prime}$ is real-analytic, viewed as a map from $\Omega \subseteq$ BMO into BMO. Moreover, its image is also an open subset of BMO, and the inverse mapping is real-analytic. (The precise definition of real-analyticity will be given in the next section.)

It may seem strange that we are using $\Psi: D \rightarrow U H P$ rather than a conformal mapping $\Phi: U H P \rightarrow D$. The reason is the latter just doesn't work out. To understand why, let's remember that $\Psi$ and $\Phi$ are not well defined, but are only defined modulo an affine transformation $z \rightarrow a z+b$ of the upper half-plane onto itself. However, if you replace $\Psi$ by $a \Psi+b$, then that merely amounts to replacing $\log h^{\prime}$ by $\log h^{\prime}+\log a$. Because BMO functions are well defined only up to an additive constant, we get that the map $\Gamma \rightarrow \log h^{\prime}$ is well defined as a map into BMO.

It doesn't work out this way if we use $\Phi$ instead of $\Psi$. Even if you add normalizations to make the map $\Gamma \rightarrow \Phi$ well defined, it is not well behaved.

Thus with this problem, as with the previous case with the Cauchy integral, we see that the symmetries of the problem tell us that BMO is the natural space, as well as how to set up the correct theorem, i.e., that the inverse of the Riemann mapping should be used.

References for these last results include [CM 2, 3, D], and the expository paper [S].

2. Some general techniques. Let me now discuss how one might deal with nonlinear functions like those discussed in the preceding section, following ideas of Coifman and Meyer.

Let $\lambda(a, f)$ be a functional that is linear in $f$ and nonlinear in $a$, where each of $a, f$, and $\lambda(a, f)$ are functions on $\mathbf{R}^{n}$. For example, take $\lambda(a, f)=$ $T_{A} f$ with $a=A^{\prime}, n=1$, and $T_{A}$ as in (1.3), or set $\lambda(a, f)=\exp (t L) f, L$ as in (1.6), $a=\left(b_{i j}\right)$. 
We say that $\lambda(a, f)$ is real-analytic in $a$ at $a=0$ as a function from $L^{\infty} \times L^{2}$ into $L^{2}$ if there is a $\delta>0$ so that

$$
\lambda(a, f)=\sum_{k=0}^{\infty} \lambda_{k}(a, f)
$$

for all $f \in L^{2}$ and all $a \in L^{\infty}$ such that $\|a\|_{\infty}<\delta$. Here each $\lambda_{k}(a, f)$ should be linear in $f$ and a homogeneous polynomial of degree $k$ in $a$. This last means that there is a $(k+1)$-linear map $\tilde{\lambda}_{k}\left(a_{1}, \ldots, a_{k}, f\right)$ such that

$$
\lambda_{k}(a, f)=\tilde{\lambda}_{k}(a, \ldots, a, f) .
$$

We also require that the power series converge absolutely in $L^{2}$, with the estimate

$$
\left\|\lambda_{k}(a, f)\right\|_{L^{2}} \leq C \delta^{-k}\|a\|_{\infty}^{k}\|f\|_{2}
$$

In the case of the operator-valued function $\tilde{C}_{\Gamma}$ defined in (1.8), we take $\lambda(a, f)=\tilde{C}_{\Gamma} f$, where $\Gamma$ has arclength parameterization $z(t)=\int_{0}^{t} e^{i a(s)} d s$, $a \in \Omega \subset$ BMO, and in (2.2) we replace $\|a\|_{\infty}$ by $\|a\|_{*}$. In the case of the Riemann mapping function, $\lambda(a)=\log h^{\prime}, h$ as in the end of the preceding section, we define real-analyticity in the same way, except now $\lambda(a)=\sum \lambda_{k}(a)$, where each $\lambda_{k}(a)$ is a homogeneous polynomial of degree $k$, and $\left\|\lambda_{k}(a)\right\|_{*} \leq C \delta^{-k}\|a\|_{*}^{k}$.

This definition may seem complicated, but in the examples it is often not so hard to write down the power series explicitly. We already saw how to do this for $T_{A}$ in (1.4).

The goal now is to find methods for proving an estimate like (2.2) so that we can sum the series (2.1).

Let's bring Fourier into this. Let $\tau_{t}$ denote the operator of translation by $t, \tau_{t} h(x)=h(x-t)$. We say that $\lambda(a, f)$ commutes with translations if

$$
\tau_{t}(\lambda(a, f))=\lambda\left(\tau_{a}, \tau_{t} f\right)
$$

for all $t$. This holds in the examples described above. This is a cute point: if you fix $A$ and view $T_{A}$ as a linear operator on $L^{2}$, then it is not a convolution operator, but if you view $T_{A} f$ as a function of both $A$ and $f$, then it does commute with translations.

If $\lambda(a, f)$ commutes with translations, then so does each $\lambda_{k}(a, f)$, and there is a representation theorem in terms of Fourier transforms. Under mild continuity assumptions on $\lambda_{k}(a, f)$, there is a distribution $\sigma_{k}(\alpha, \zeta)=$ $\sigma_{k}\left(\alpha_{1}, \alpha_{2}, \ldots, \alpha_{k}, \zeta\right)$ such that

$$
\lambda_{k}(a, f)(x)=\int_{\mathbf{R}^{n}} \cdots \int_{\mathbf{R}^{n}} e^{2 \pi i x\left(\sum_{j} \alpha_{j}+\zeta\right)} \sigma_{k}(\alpha, \zeta) \hat{a}\left(\alpha_{1}\right) \cdots \hat{a}\left(\alpha_{k}\right) \hat{f}(\zeta) d \alpha d \zeta .
$$

We call $\sigma_{k}(\alpha, \zeta)$ the symbol of $\lambda_{k}$, and it is uniquely determined by $\lambda_{k}$ if it is symmetric in the $\alpha_{j}$ 's.

When $k=0, \lambda_{0}(a, f)$ does not depend on $a$, and the above representation reduces to the well-known result that a convolution operator $T$ can be 
written as $(T f)^{\wedge}(\zeta)=m(\zeta) \hat{f}(\zeta)$ for some $m(\zeta)$. In this case $T$ is bounded on $L^{2}$ iff $m \in L^{\infty}$, by Plancherel's theorem.

When $k>0$ we do not get useful estimates from Plancherel. We do, however, have the following result of Coifman and Meyer [CM1].

Theorem 2.3. Suppose $\sigma_{k}(\alpha, \zeta)$ is smooth away from the origin and satisfies

$$
\left|\left(\frac{\partial}{\partial \alpha}\right)^{p}\left(\frac{\partial}{\partial \zeta}\right)^{q} \sigma_{k}(\alpha, \zeta)\right| \leq C(p, q)(|\alpha|+|\zeta|)^{-|p|-q} \text { for all } p, q .
$$

Then $\left\|\lambda_{k}(a, f)\right\|_{2} \leq C\|a\|_{\infty}^{k}\|f\|_{2}$, where $C$ depends on $k$ and the constants $C(p, q)$ above.

This result is really quite fundamental. It and its proof techniques give a lot of insight into the way multilinear operators should be analyzed. I shall give a simple example of this at the end of the section.

There are two main problems with this theorem. The first is that the symbol is usually not so smooth in the examples. This turns out not to be too serious - the examples can usually be dealt with using straightforward technical refinements of the theorem or by adding comparatively simpler ad-hoc arguments.

The second problem is more serious. We want to use Theorem 2.3 for summing series like (2.1), with estimates as in (2.2). Unfortunately, Theorem 2.3 does not give enough control on the norm of $\lambda_{k}$ as a function of $k$ to get (2.2). However, the following result of David and Jorné [DJ] can often be used to get estimates like (2.2).

The $T(1)$ Theorem. Let $T: C_{0}^{\infty}\left(\mathbf{R}^{n}\right) \rightarrow C_{0}^{\infty}\left(\mathbf{R}^{n}\right)^{\prime}$ be linear and continuous, and suppose that $T$ is associated to a kernel $K(x, y)$ such that $K(x, y)=-K(y, x)$ and

$$
\begin{gathered}
|K(x, y)| \leq C|x-y|^{-n} \\
\left|\nabla_{x} K(x, y)\right|+\left|\nabla_{y} K(x, y)\right| \leq C|x-y|^{-n-1} .
\end{gathered}
$$

Then $T$ extends to a bounded operator on $L^{2}\left(\mathbf{R}^{n}\right)$ if $T(1) \in \operatorname{BMO}\left(\mathbf{R}^{n}\right)$.

The connection between this result and estimates like (2.2) is not obvious, and I'll explain that after I've explained some of the definitions.

Here $C_{0}^{\infty}$ means the space of $C^{\infty}$ functions with compact support. To say that $T$ is associated to $K(x, y)$ means that if $f, g \in C_{0}^{\infty}\left(\mathbf{R}^{n}\right)$ have disjoint support, then

$$
\langle T f, g\rangle=\int_{\mathbf{R}^{n}} \int_{\mathbf{R}^{n}} g(x) K(x, y) f(y) d y d x .
$$

This holds if $T f(x)$ is given by a principal value integral with $K(x, y)$.

There is a version of this theorem when $K$ is not antisymmetric, but then an additional mild continuity assumption is needed, and $T(1) \in \mathrm{BMO}$ is replaced by $T(1), T^{*}(1) \in \mathrm{BMO}$. 
$\mathrm{BMO}(\mathbf{R})$ was defined at the end of the first section. (See (1.9).) $\mathrm{BMO}\left(\mathbf{R}^{n}\right)$ is defined in the same way, except that intervals are replaced by balls.

If $T$ is bounded on $L^{2}$ and is associated to a kernel $K(x, y)$ that satisfies (2.4) and (2.5), then in general $T$ does not map $L^{\infty}$ into $L^{\infty}$. Indeed, $T(f)$ will not even be well defined if $f$ is merely bounded, because (2.4) is not strong enough to make the integral converge at $\infty$. However, one can show that $T(f)$ is defined modulo additive constants, using (2.5). Thus it is natural that $T$ actually maps $L^{\infty}$ into BMO. Moreover, BMO is the smallest space of functions for which this result holds.

Let me illustrate how the $T(1)$ theorem can be used to obtain estimates like (2.2) through an example, namely, the series for $T_{A}$ in (1.4).

If $A$ is Lipschitz, then $A^{\prime} \in L^{\infty}$, and one can easily check that the kernel $(A(x)-A(y))^{k} /(x-y)^{k+1}$ of $T_{k}=T_{k}(A)$ satisfies the hypotheses of the $T(1)$ theorem. An integration by parts computation gives

$$
T_{k}(1)=T_{k-1}\left(A^{\prime}\right) \text {. }
$$

Hence, if $T_{k-1}$ is bounded on $L^{2}$, so that $T_{k-1}$ also maps $L^{\infty}$ into BMO, then $T_{k}(1) \in \mathrm{BMO}$, and $T_{k}$ is also bounded on $L^{2}$, by the $T(1)$ theorem.

This induction argument gives the estimate

$$
\left\|T_{k}(A) f\right\|_{2} \leq C^{k}\left\|A^{\prime}\right\|_{\infty}^{k}\|f\|_{2} \text {. }
$$

This allows the series (1.4) to be summed when $\left\|A^{\prime}\right\|_{\infty}<C^{-1}$, and to obtain Theorem 1.5 in that case. To prove that the radius of convergence is 1 , and to prove Theorem 1.5 in the general case, requires more refined arguments.

This example is quite typical of how the $T(1)$ theorem is used. Given a $(k+1)$-linear functional $L\left(a_{1}, \ldots, a_{k}, f\right)$, the $T(1)$ theorem reduces the problem of getting estimates for this to the task of controlling an object like $L\left(a_{1}, \ldots, a_{k}, 1\right)$. In other words, the $T(1)$ theorem allows one to go from a $(k+1)$-linear functional to a $k$-linear functional, which sets up a natural induction procedure.

In the next section I shall discuss some aspects of the proof of the $T(1)$ theorem, and also an extension of it.

I'd like to end this section with an example to illustrate how one analyzes multilinear operators on the symbol side, using Theorem 2.3.

Let $f, g$ be two functions on $\mathbf{R}$. Setting $D=d / d x$, we have the familiar Leibnitz rule

$$
D(f g)=(D f) g+f(D g) \text {. }
$$

This formula no longer holds if we replace $D$ by some power of $D$. Even for integer powers of $D$ we still get extra terms.

Let's ask for a weaker form of the Leibnitz rule for powers of $D$. Given $s>0$, can we find nice bilinear operators $B_{1}(f, g), B_{2}(f, g)$ such that

$$
|D|^{s}(f g)=B_{1}\left(|D|^{s} f, g\right)+B_{2}\left(f,|D|^{s} g\right) ?
$$

Here $|D|^{s}$ is defined by

$$
\left(|D|^{s} f\right)^{\sim}(\zeta)=|\zeta|^{s} \hat{f}(\zeta) .
$$


By "nice" we mean in particular that $B_{1}(\cdot, \cdot)$ and $B_{2}(\cdot, \cdot)$ should satisfy $L^{p}$ estimates like those for $B(f, g)=f g$ given by Hölder's inequality.

Let us compute on the Fourier transform side. By definitions,

$$
f(x) g(x)=\iint^{2 \pi i(\zeta+\eta)} \hat{f}(\zeta) \hat{g}(\eta) d \zeta d \eta
$$

and $|D|^{s}(f g)(x)=\iint^{2 \pi i x(\zeta+\eta)}|\zeta+\eta|^{s} \hat{f}(\zeta) \hat{g}(\eta) d \zeta d \eta$. To get (2.6), we want the symbols $\sigma_{1}(\zeta, \eta)$ and $\sigma_{2}(\zeta, \eta)$ of $B_{1}(\cdot, \cdot)$ and $B_{2}(\cdot, \cdot)$ to satisfy

$$
|\zeta+\eta|^{s}=\sigma_{1}(\zeta, \eta)|\zeta|^{s}+\sigma_{2}(\zeta, \eta)|\eta|^{s}
$$

and we want these symbols to be reasonably well behaved.

We use a suitable cut-off function. Let $\phi(\zeta, \eta)$ be such that $\phi(\zeta, \eta)=0$ if $|\zeta| \leq \frac{1}{2}|\eta|, \phi(\zeta, \eta)=1$ if $2|\eta| \leq|\zeta|$, and

$$
\left|\partial_{\zeta}^{q} \partial_{\eta}^{q} \phi(\zeta, \eta)\right| \leq C(p, q)(|\zeta|+|\eta|)^{-p-q} .
$$

Thus $\phi(\zeta, \eta)$ vanishes when $\zeta$ is small compared to $\eta$. (We can also choose $\phi$ to be homogeneous of degree 0 .)

Set

$$
\begin{aligned}
& \sigma_{1}(\zeta, \eta)=|\zeta+\eta|^{s}|\zeta|^{-s} \phi(\zeta, \eta) \\
& \sigma_{2}(\zeta, \eta)=|\zeta+\eta|^{s}|\eta|^{-s}(1-\phi(\zeta, \eta)) .
\end{aligned}
$$

Then (2.7) holds automatically, and the specific choice of $\phi$ keeps $\sigma_{1}$ and $\sigma_{2}$ from blowing up.

If $s$ is an even integer, Theorem 2.3 applies to the bilinear operators $B_{1}$ and $B_{2}$, and the proof of Theorem 2.3 gives much more information (other $L^{p}$ estimates, in particular). Even if $s$ is not an even integer, the method of proof of Theorem 2.3 permits one to show that $B_{1}$ and $B_{2}$ are well behaved.

This example is very simple, but the computations used are quite typical.

3. Some aspects of Littlewood-Paley theory. Littlewood-Paley theory provides an important tool for analyzing operators and functions. To get an idea of how it works, let us consider a specific problem.

Let $T$ be as in the $T(1)$ theorem, but with $T(1)=0$. Let us see why $T$ has to be bounded on $L^{2}$. We restrict ourselves to the real line for simplicity. An example of such an operator is the Hilbert transform

$$
H f(x)=p v \int_{-\infty}^{\infty} \frac{f(y)}{x-y} d y .
$$

A general method for analyzing an operator $T$ is to choose an orthonormal basis $\left\{e_{i}\right\}$ for $L^{2}$ and to express $T$ in terms of this basis:

$$
T f=\sum_{i j}\left\langle T e_{i}, e_{j}\right\rangle\left\langle f, e_{i}\right\rangle e_{j}
$$

Information about the matrix $\left\langle T e_{i}, e_{j}\right\rangle$ can be used to get information about $T$, for example, the boundedness of $T$ on $L^{2}$. 
To make this work, we need to choose the basis $\left\{e_{i}\right\}$ carefully, so that we can compute $\left\langle T e_{i}, e_{j}\right\rangle$ and also get something that we can work with. We would like to choose the basis $\left\{e_{i}\right\}$ so that $T$ is almost diagonalized, in some reasonable sense.

One choice for an orthonormal basis for $L^{2}$ is given by the Fourier transform. Of course this isn't an orthonormal basis in the usual sense, but all that means is that we have to replace sums with integrals. This works in the case of the Hilbert transform, because it is diagonalized by the Fourier transform, and $L^{2}$-estimates follow from Plancherel's theorem.

However, if $T$ is as in the $T(1)$ theorem, but $T$ is not a convolution operator, then the Fourier transform approach doesn't work. For this problem, the Fourier transform is too sensitive to the algebra and not sensitive enough to the geometry.

A better choice of basis for $L^{2}(\mathbf{R})$ is the Haar basis, defined as follows. Let $\Delta$ denote the collection of dyadic intervals on $\mathbf{R}$, i.e., the intervals of the form $\left[k 2^{j},(k+1) 2^{j}\right)$ for $j, k \in \mathbf{Z}$. Given $I \in \Delta$, define the associated Haar function $h_{I}$ on $\mathbf{R}$ by $h_{I}(x)=0$ if $x \notin I, h_{I}(x)=|I|^{-1 / 2}$ if $x$ lies in the left half of $I, h_{I}(x)=-|I|^{-1 / 2}$ if $x$ lies in the right half of $I$. (If $I=\left[k 2^{j},(k+1) 2^{j}\right)$, then the left and right halves of $I$ are given by

$$
\left[k 2^{j},\left(k+\frac{1}{2}\right) 2^{j}\right) \text { and }\left[\left(k+\frac{1}{2}\right) 2^{j},(k+1) 2^{j}\right),
$$

respectively.)

The definition of $h_{I}$ implies that $\int\left|h_{I}\right|^{2}=1$ for all $I$. It is not hard to show that $h_{I}, I \in \Delta$, forms an orthonormal sequence in $L^{2}(\mathbf{R})$. This uses the basic fact that if $I, J \in \Delta$, then either $I \subseteq J, J \subseteq I$, or $I \cap J=\varnothing$. Moreover, $h_{I}, I \in \Delta$, forms an orthonormal basis for $L^{2}(\mathbf{R})$.

In a very real sense, operators $T$ that satisfy $T(1)=0$ and the assumptions of the $T(1)$ theorem are almost diagonalized by the $h_{I}$ 's. One can show that the matrix entry $\left\langle T h_{I}, h_{J}\right\rangle$ gets small as you move away from the diagonal, i.e., when $I$ and $J$ are far away from each other, or when one is much smaller than the other. A precise version of this fact can be used to prove that $T$ is bounded on $L^{2}$. For example, Schur's criterion says that $T$ is bounded on $L^{2}(\mathbf{R})$ if

$$
\sup _{I} \sum_{J}\left|\left\langle T h_{I}, h_{J}\right\rangle\right|+\sup _{J} \sum_{I}\left|\left\langle T h_{I}, h_{J}\right\rangle\right|<\infty .
$$

One can check that (3.1) holds under our assumptions on $T$.

The $h_{I}$ 's form a good basis for functions on $\mathbf{R}$ in other respects as well. For example, $\left\{h_{I}\right\}$ forms an unconditional basis for $L^{p}(\mathbf{R}), 1<p<\infty$. (It is known that $L^{1}$ and $L^{\infty}$ don't have unconditional bases.) This means that every $f \in L^{p}(\mathbf{R})$ has a unique expansion of the form $f=\sum \alpha_{I} h_{I}$, and that the $L^{p}$ norm of $f$ depends essentially only on the size of the $\alpha_{I}$ 's (and not on oscillation properties, for instance). In this case of the $h_{I}$ 's one has $\alpha_{I}=\left\langle f, h_{I}\right\rangle$, and the $L^{p}$ norm of $f$ is equivalent in size to $\|A(f)\|_{p}$, where

$$
A(f)(x)=\left(\sum_{I \ni x}\left|\alpha_{I}\right|^{2}|I|^{-1}\right)^{1 / 2} .
$$


By contrast, there is no reasonable characterization of the Fourier transform of $L^{p}$ functions.

The basis $\left\{h_{I}\right\}$ respects the geometry of $\mathbf{R}$ much better than the Fourier transform. In this regard it is very helpful that the $h_{I}$ 's are localized in space-i.e., supported in the interval $I$-and that they also don't oscillate much. The $h_{I}$ 's do have an important weakness, which is that they are not very smooth. There is a fairly simple alternate approach that doesn't have this problem, but let me invert the order of history and first describe an elegant approach of Strömberg [Sb]. (See also [M,1, 2 and LM].)

Strömberg showed that, given $m$, one can find a function $\psi$ so that $\psi$ and its first $m$ derivatives decay exponentially fast at $\infty$, and such that if

$$
\psi_{I}(x)=|I|^{-1 / 2} \psi\left(2^{-j}\left(x-k 2^{j}\right)\right),
$$

$I=\left[k 2^{j},(k+1) 2^{j}\right)$, then $\left\{\psi_{I}\right\}, I \in \Delta$, is an orthonormal basis for $L^{2}(\mathbf{R})$. Notice that the $h_{I}$ 's can be obtained from $h=h_{[0,1]}$ exactly as in (3.3).

Unlike the $h_{I}$ 's, $\psi_{I}$ is not supported in $I$. However, $\psi_{I}$ is mostly concentrated near $I$; as $x$ moves away from $I, \psi_{I}(x)$ goes to 0 . Although $\psi_{I}$ is not piecewise constant like $h_{I}$ is, its smoothness means that it is roughly constant on invervals that are small compared to $I$. Like $h_{I}, \psi_{I}$ satisfies $\int \psi_{I}=0$.

The family $\left\{\psi_{I}\right\}$ forms an unconditional basis for $L^{p}(\mathbf{R}), 1<p<\infty$ and also for Sobolev spaces, Lipschitz and Besov spaces, Hardy spaces, and BMO. The Haar functions do not work in these other cases because of their lack of smoothness. Unconditional bases for these spaces were known previously, but they were not as elegant as $\left\{\psi_{I}\right\}$.

Like the Haar basis, $\left\{\psi_{I}\right\}$ "almost diagonalizes" operators $T$ that satisfy the hypotheses of the $T(1)$ theorem and also $T(1)=0$. In fact, $\left\{\psi_{I}\right\}$ works better than the Haar functions; the estimates for $\left\langle T \psi_{I}, \psi_{J}\right\rangle$ are better than those for $\left\langle T h_{I}, h_{J}\right\rangle$, because of the discontinuities of the $h_{I}$ 's.

There are other applications of the bases $\left\{\psi_{I}\right\}$ and the methods used to construct them, but I shall not say more about that. The upcoming book by Meyer will be the basic reference. Papers currently in existence include [Sb, M1, 2 and LM], and further references can be found in these.

I should point out that the Haar functions and the $\psi_{I}$ 's have suitable versions in $\mathbf{R}^{n}$.

Let me describe now an older and more straightforward method of fixing the nonsmoothness of the Haar functions.

Let $\psi(x)$ be any real-valued Schwartz function that is radial and not identically zero, and that satisfies $\int \psi=0$. Set $\psi_{t}(x)=t^{-n} \psi(x / t)$ for $t>0$. Then there is a $c \neq 0$ such that

$$
f=c \int_{0}^{\infty} \psi_{t} * \psi_{t} * f \frac{d t}{t}
$$

for all $f$ on $\mathbf{R}^{n}$. This is easily checked using the Fourier transform. The identity (3.4) is often called a Calderón reproducing formula. 
Let us rewrite (3.4) in a more suggestive way. Setting $\psi_{t, u}(x)=\psi_{t}(x-u)$, we have

$$
f=c \int_{0}^{\infty} \int_{\mathbf{R}^{n}} \psi_{t, u}\left\langle f, \psi_{t, u}\right\rangle d u \frac{d t}{t} .
$$

We think of this as being analogous to the formula for expanding $f$ in terms of an orthonormal basis $\left\{\boldsymbol{e}_{i}\right\}$,

$$
f=\sum_{i}\left\langle f, e_{i}\right\rangle e_{i}
$$

with the index $i$ replaced by $(u, t) \in \mathbf{R}_{+}^{n+1}$, and the sum replaced by the integral.

The expansion (3.5) satisfies $L^{2}$-estimates similar to those for (3.6). Analogous to $\|v\|=\left(\sum\left|\left\langle v, e_{i}\right\rangle\right|^{2}\right)^{1 / 2},\left\|\sum \alpha_{i} e_{i}\right\| \leq\left(\sum\left|\alpha_{i}\right|^{2}\right)^{1 / 2}$, we have

$$
\|f\|_{2}=a\left(\int_{0}^{\infty} \int_{\mathbf{R}^{n}}\left|\left\langle f, \psi_{t, u}\right\rangle\right|^{2} \frac{d u d t}{t}\right)^{1 / 2}
$$

and

$$
\left\|\int_{0}^{\infty} \int_{\mathbf{R}^{n}} \psi_{t, u} G(u, t) d u \frac{d t}{t}\right\|_{2} \leq C\left(\int_{0}^{\infty} \int_{\mathbf{R}^{n}}|G(u, t)|^{2} d u \frac{d t}{t}\right)^{1 / 2}
$$

for some $a \neq 0, C<\infty$, and for all functions $f$ on $\mathbf{R}^{n}, G$ on $\mathbf{R}_{+}^{n+1}$.

Just as the Haar functions and Strömberg's bases were unconditional bases for other function spaces, there are also analogues of (3.7) and (3.8) for $L^{p}$ spaces, BMO, Lipschitz spaces, etc. (In these cases, the version of (3.7) will be an equivalence of norms.)

Similarly, if $T$ satisfies the hypotheses of the $T(1)$ theorem and also $T(1)=0$, then $T$ is almost diagonalized by the expansion (3.5). Just like (3.1), we have

$$
T f=\int_{0}^{\infty} \int_{\mathbf{R}^{n}} \int_{0}^{\infty} \int_{\mathbf{R}^{n}}\left\langle T \psi_{t, u}, \psi_{s, v}\right\rangle\left\langle f, \psi_{t, u}\right\rangle \psi_{s, v} d v \frac{d s}{s} d u \frac{d t}{t}
$$

Again the "matrix entries" $\left\langle T \psi_{t, u}, \psi_{s, v}\right\rangle$ tend to 0 as $(t, u)$ and $(s, v)$ move away from each other, and the boundedness of $T$ on $L^{2}$ can be derived from a precise version of this fact and Schur's lemma.

An important respect in which (3.5) is not like an orthonormal expansion is that there is no uniqueness: given $f$, there are many functions $F(u, t)$ on $\mathbf{R}_{+}^{n+1}$ such that

$$
f=\int_{0}^{\infty} \int_{\mathbf{R}^{n}} \psi_{t, u} F(u, t) d u \frac{d t}{t} .
$$

For many applications, though, this lack of uniqueness does not matter, and (3.5), (3.7), and (3.8) are already good enough.

The $\psi_{t, u}$ 's respect the geometry in much the same way the $h_{I}$ 's and $\psi_{I}$ 's do. The $\psi_{t, u}$ 's are localized in space; $\psi_{t, u}$ is roughly concentrated in the ball $B(u, t)=\{x:|x-u| \leq t\}$, in the sense that $\psi_{t, u}(x)$ goes to 0 as $|x-u|$ 
becomes large compared to $t$. If we choose the original function $\psi$ to be supported in $B(0,1)$, then we would actually have supp $\psi_{t, u} \subseteq B(u, t)$.

Also, the $\psi_{t, u}$ 's are smooth at the scale of $t$. It is easy to compute the gradient of $\psi_{t, u}$ and check that $\psi_{t, u}$ is almost constant on balls with radius that is small compared to $t$.

This last property can be rephrased in terms of Fourier transforms by saying that $\hat{\psi}_{t, u}$ is roughly concentrated in the annulus

Indeed,

$$
\left\{\zeta: \frac{1}{10} t^{-1}<|\zeta|<10 t^{-1}\right\}
$$

$$
\hat{\psi}_{t, u}(\zeta)=e^{i \zeta \cdot u} \hat{\psi}(t \zeta)
$$

and this tends to zero as $t \zeta$ tends to $\infty$ or 0 . This last holds because $\hat{\psi}(0)=$ $\int \psi=0$. We could have chosen $\psi$ so that $\operatorname{supp} \psi \subseteq\left\{\frac{1}{10}<|\zeta|<10\right\}$, which would give

$$
\operatorname{supp} \hat{\psi}_{t, u} \subseteq\left\{\frac{1}{10} t^{-1}<|\zeta|<10 t^{-1}\right\} .
$$

Thus the $\psi_{t, u}$ 's are localized both in the space and frequency variables, and that is really what makes the whole thing go.

I have not said anything so far about how the $T(1)$ theorem is proved when $T(1) \neq 0$, nor how to prove Theorem 2.3. The analysis in these cases requires more tools. A principal ingredient is a certain class of operators (often called paraproducts) introduced by Coifman and Meyer. These operators act as basic building blocks; many of the operators that arise in practice can be written in terms of paraproducts together with other terms that are simpler. Paraproducts have also been used by Bony [B] in connection with regularity problems for nonlinear PDE.

I'd like to discuss now a generalization of the $T(1)$ theorem and a related generalization of Littlewood-Paley theory. Yves Meyer posed the question of whether there might be a version of the $T(1)$ theorem in which the function 1 is allowed to be replaced by any $b \in L^{\infty}$ such that $\operatorname{Re} b \geq \delta$ on $\mathbf{R}^{n}$ for some $\delta>0$. An example of an operator to which such a theorem would apply is the Cauchy integral on a Lipschitz graph: the operator $T_{A}$ in (1.3) satisfies $T_{A}(b)=0$, with $b=1+i A^{\prime}$, because of Cauchy's theorem.

When I first heard of this question, my reaction was "Why should that be true?" and "How could one possibly prove it?" Thus it came as quite a surprise to me when Alan McIntosh and Meyer [McM] showed that it was true, at least in the case $T(b)=0$.

This beautiful theorem of McIntosh and Meyer was extended in [DJS]. In particular, the class of admissible functions $b$ was enlarged so that there are now necessary and sufficient conditions on $b$ for the theorem to hold.

The method of proof in [DJS] was to build a version of LittlewoodPaley theory that is adapted to the given function $b$. In other words, the set-up is adjusted to the measure $b(x) d x$ instead of Lebesgue measure. Let me explain what this means.

We would like to have analogues $\tilde{\psi}_{t, u}$ of the functions $\psi_{t, u}$ that satisfy $\int \tilde{\psi}_{t, u}(x) b(x) d x=0$ instead of $\int \psi_{t, u}=0$. The size and smoothness 
properties of $\tilde{\psi}_{t, u}$ should be the same as those for $\psi_{t, u}$, that is, $\tilde{\psi}_{t, u}$ should be smooth at the scale of $t$, and $\tilde{\psi}_{t, u}(x)$ should decay as $|x-u|$ becomes large compared to $t$.

We also want the $\tilde{\psi}_{t, u}$ 's to satisfy properties similar to (3.5), (3.7), and (3.8), i.e.,

$$
f(x)=c \int_{0}^{\infty} \int_{\mathbf{R}^{n}} \tilde{\psi}_{t, u}(x) b(u) F(u, t) d u \frac{d t}{t}
$$

with $F(u, t)=\int \tilde{\psi}_{t, u}(y) b(y) f(y) d y$, and

$$
\int_{0}^{\infty} \int_{\mathbf{R}^{n}}|F(u, t)|^{2} \frac{d u d t}{t} \leq C\|f\|_{2}^{2},
$$

as well as (3.8) with $\psi_{t, u}$ replaced by $\tilde{\psi}_{t, u}$.

If we could build $\tilde{\psi}_{t, u}$ satisfying these properties, then we could prove the $T(b)$ theorem (=the $T(1)$ theorem with 1 replaced by $b$ ) in the same way as the $T(1)$ theorem. For example, the argument outlined before for the case $T(1)=0$ would go over with only cosmetic changes.

Building the $\tilde{\psi}_{t, u}$ 's is a substantially more difficult task than in the case $b=1$. For one thing, the identity (3.5) came from a Fourier transform calculation that is not available when $b$ is not constant. This is already an issue when $b$ is real and positive, or if you want a version of the $T(1)$ theorem on a space where there is no Fourier transform.

In the case where $b>0$, this problem was resolved by Coifman, and his solution is described in [DJS]. However, Coifman's approach used heavily the fact that $b$ is positive; the estimates used Hilbert space tricks that break down when $b$ is complex. This obstacle was overcome in [DJS], and $\tilde{\psi}_{t, u}$ 's were constructed with the necessary properties. (I'm cheating here slightly; the correct version is a bit more complicated than what's written above.)

4. Other expository papers on related topics. The natural place to start is [CM4]. There is some overlap between that paper and this one, but the symmetric difference of the two is large. In the overlapping parts, [CM4] gives more details.

I am very fond of the paper [C4] by Calderón. A hugh amount of progress has occurred since then, but he gives a very lucid explanation of some reasons for the interest in the Cauchy integral on Lipschitz graphs and in the estimates for the Calderón commutators, i.e., the operators $T_{k}(A)$ in (1.4).

One reason for Calderón's interest in these operators came from his interest in building algebras of singular integral operators for studying problems in PDE. The pseudodifferential calculus gives an alternate approach to similar problems with a more powerful symbolic calculus, but at the expense of needing the coefficients of the given differential operators to be smooth. Calderón's approach gives a less powerful calculus, but with minimal smoothness requirements on the coefficients. The $L^{2}$-boundedness of the first commutator $T_{1}(A)$ is an important ingredient for that.

Boundary value problems on Lipschitz domains gives another class of PDE problems related to these topics. A classical approach is the method 
of layer potentials, in which a boundary value problem is reduced to solving an integral equation on the boundary. These integral equations involve singular integral operators whose boundedness on $L^{p}$ follows from the theorems of Calderón and Coifman, McIntosh and Meyer. This implies that the integral equations are well defined on $L^{p}$, but solving the integral equations is another matter. A good reference for this is the survey paper of Kenig [K].

The complex-variable aspects of these topics are discussed in more detail in [S]. The work of Coifman and Meyer on the real-analyticity of the Cauchy integral operator and the Riemann mapping on the space of chordarc curves is described, as well as the relationship between their work and quasiconformal mappings and $\bar{\partial}$.

The discussion of Littlewood-Paley Theory given in $\S 3$ is quite limited in scope. The reader would be well-served by reading the expository paper [Sn1] by Stein to learn about other aspects of the subject, and also its historical development. Another good reference is the monograph [Sn2].

\section{REFERENCES}

[B] J. M. Bony, Calcul symbolique et propagation des singularités pour les équations aux dérivées partielles non linéares, Ann. Sci. École Norm. Sup. (4) 14 (1981), 209-246.

[C1] A. P. Calderón, Commutators of singular integral operators, Proc. Nat. Acad. Sci. U.S.A. 53 (1965), 1092-1099.

[C2] - Algebras of singular integral operators, Proc. Sympos. Pure Math., vol. 10, Amer. Math. Soc., Providence, R. I., 1966, pp. 18-55.

[C3] _ Cauchy integrals on Lipschitz curves and related operators, Proc. Nat. Acad. Sci. U.S.A. 74 (1977), 1324-1327.

[C4] _ Commutators, singular integrals on Lipschitz curves, and applications, Proc. Internat. Congr. Math. (Helsinki, 1978), Acad. Sci. Fennica, Helsinki, 1980, pp. 85-96.

[CM1] R. Coifman and Y. Meyer, Au-delà des opérateurs pseudo-différentiels, Astérisque 57 (1978).

[CM2] __, Une généralisation du théorème de Calderón sur I'integrale de Cauchy, Fourier Analysis, Proc. Sem. (El Escorial, 1979), (M. de Guzmàn and I. Peral, eds.) Asoc. Mat. Española, Madrid, 1980.

[CM3] _ Lavrentiev's curves and conformal mapping, Institut Mittag-Leffler, Report No. $5,1983$.

[CM4] _ Non-linear harmonic analysis, operatory theory, and PDE, Beijing Lectures in Harmonic Analysis, (E. M. Stein, ed.), Ann. of Math. Studies No. 12, Princeton Univ. Press, Princeton, N.J., 1986.

[CDM] R. Coifman and D. G. Deng, and Y. Meyer, Domaine de la racine carrée de certains opérateurs différentiels accrétifs, Ann. Inst. Fourier (Grenoble) 33 (1983), 123-134.

[CMM] R. Coifman, A. McIntosh and Y. Meyer, L'integrale de Cauchy définit un opérateur borné sur $L^{2}$ pour les courbes lipschitziennes, Ann. of Math. (2) 116 (1982), 361388.

[D] G. David, Thése de troisiéme cycle, Université de Paris XI, 91405 Orsay, France.

[DJ] G. David and J. L. Journé, A boundedness criterion for generalized CalderonZygmund operators, Ann. of Math. (2) 120 (1984), 371-397.

[DJS] G. David, J. L. Journé, and S. Semmes, Opérateurs de Calderón-Zygmund, fonctions para-accétives, et interpolation, Rev. Mat. Ibero-Americana 1 (4) (1984), 1-56.

[FJK1] E. Fabes, D. Jerison, and C. Kenig, Multilinear Littlewood-Paley estimates with applications to partial differential equations, Proc. Nat. Acad. Sci. U.S.A. 79 (1982), 57465750. 
[FJK2] _ Necessary and sufficient conditions for absolute continuity of elliptic-harmonic measure, Ann. of Math. (2) 119 (1984), 121-141.

[FJK3] _ Multilinear square functions and partial differential equations, Amer. J. Math. 107 (1985), 1325-1368.

[K] C. Kenig, Elliptic boundary value problems on Lipschitz domains, Beijing Lectures in Harmonic Analysis (E. M. Stein, ed.) Ann. of Math. Studies No. 112, 1986.

[KM] C. Kenig and Y. Meyer, Kato's square roots of accretive operators and Cauchy kernels on Lipschitz graphs are the same, Recent progress in Fourier analysis, Proceedings of the Conference at El Escorial, Spain (1983), pp. 123-143, North-Holland Math. Stud. 111.

[LM] P. G. Lemarié and Y. Meyer, Ondelettes et bases hilbertiennes, Rev. Mat. IberoAmericana 2 (1986), 1-19.

[M1] Y. Meyer, Principe d'incertitude, bases hilbertiennes, et algèbres d'operateurs, Seminaire Bourbaki, Feb. 1986, no. 662.

[M2] _ Wavelets and operators, preprint. CEREMADE, Université de Paris, Dauphine.

[McM] A. McIntosh and Y. Meyer, Algèbre d'opérateurs définis par des intégrales singuliéres, C. R. Acad. Sci. Paris 301 (1985), 395-397.

[S] S. Semmes, The Cauchy integral, chord-arc curves, and quasiconformal mappings, The Bieberbach Conjecture, Proceedings of the Symposium on the occasion of the proof, (A. Baernstein, et. al. eds.) Math. Surveys, no. 21, Amer. Math. Soc., Providence, R. I., 1986.

[Sb] J. O. Strömberg, A modified Franklin system and higher order spline systems on $\mathbf{R}^{n}$ as unconditional bases for Hardy spaces, Conference on Harmonic Analysis in Honor of Antoni Zygmund, Vol. II, pp. 475-493, (W. Beckner et. al., eds.), Wadsworth math. Series.

[Sn1] E. Stein, The development of square functions in the work of A. Zygmund, Bull. Amer. Math. Soc. (N.S.) 7 (1982), 359-376.

[Sn2] _ Topics in harmonic analysis related to the Littlewood-Paley theory, Ann. of Math. Studies, vol. 63, Princeton Univ. Press, Princeton, N. J., 1970.

Department of Mathematics, Rice University, Houston, TeXas 77251 